\title{
Long noncoding RNA and mRNA profiling in MDA-MB-23I cells following RNAi-mediated knockdown of SIRT7
}

This article was published in the following Dove Press journal:

OncoTargets and Therapy

24 October 2017

Number of times this article has been viewed

\author{
Kun-Lin Chen \\ Lian Li \\ Yi-Ru Wang \\ Cheng-Min Li \\ Tarig Mohammed Badri \\ Gen-Lin Wang \\ Animal Genetics, Breeding and \\ Reproduction Department, College \\ of Animal Science and Technology, \\ Nanjing Agricultural University, \\ Nanjing, People's Republic of China
}

\begin{abstract}
Breast cancer is one of the most common malignant cancers among women and a major clinical obstacle. Although studies have reported the abnormal expression of SIRT7 in breast cancer, whether the function of SIRT7 regulates the expression of long noncoding RNAs (lncRNAs) in breast cancer remains unknown. We aimed to determine the differential expressions of mRNAs and lncRNAs associated with SIRT7 and understand the regulatory mechanism of SIRT7 in breast cancer. RNA sequencing was performed to explore the transcriptome in MDA-MB-231 cells after SIRT7 depletion, and a total of 50,634 different transcripts were identified. In comparison with the negative control, siSIRT7 groups showed 240 differentially expressed mRNAs and 26 differentially expressed lncRNAs. Gene ontology analysis revealed that the differentially expressed mRNAs mainly regulated DNA replication, CXCR chemokine receptor binding, and maturation of large subunit rRNA from tricistronic rRNA transcript, nucleoplasm, mitochondrion, and $\mathrm{NAD}^{+}$ADP-ribosyltransferase activity. Kyoto Encyclopedia of Genes and Genomes analysis showed that the differentially expressed mRNAs were mainly involved in pathways associated with MAPK signaling pathway, tumor necrosis factor signaling pathway, hepatitis B, and cancer. Moreover, the target genes of the differentially expressed lncRNAs mainly regulated the carboxylic acid metabolic processes and were involved in glycolysis pathway. The mRNA-lncRNA coexpression network comprised 186 mRNAs and $23 \mathrm{lncRNAs}$. Our results provide essential data regarding differentially expressed lncRNAs and mRNAs after the depletion of SIRT7 in breast cancer cells, which may be useful to elucidate the role of SIRT7 in breast cancer development.
\end{abstract}

Keywords: SIRT7, breast cancer cell, lncRNA, mRNA, RNA-Seq

\section{Introduction}

Sirtuins are a highly conserved family of $\mathrm{NAD}^{+}$-dependent deacetylases present in all eukaryotes and play various roles in metabolism, genome stability, stress response, and life span. ${ }^{1,2}$ There are seven sirtuins (SIRT1-7) in mammals, which are localized in different cellular compartments and exert their functions on a wide range of protein substrates. ${ }^{3}$ Sirtuins have recently gained research attention due to their effects on tumorigenesis. Some sirtuins play critical roles in carcinogenesis by promoting tumor cell growth and inhibiting cell apoptosis, while others have the opposite effect on tumorigenesis. ${ }^{4,5}$

SIRT7 is the only sirtuin that is predominantly distributed in the nucleolus and known for its role in maintaining deacetylated state of $\mathrm{H} 3 \mathrm{~K} 18$ at promoters of some tumor genes. ${ }^{6}$ Furthermore, SIRT7 plays essential roles in chromatin regulation, cellular transformation programs, and tumor formation by mediating 
H3K18 deacetylation. ${ }^{7}$ Thus, the inhibition of H3K18 deacetylation may induce malignant transformation and highly malignant cancer. ${ }^{8,9}$ The SIRT7-ELK4 connection is also essential for cancer development. It was previously reported that the depletion of ELK4 may increase H3K 18 acetylation specifically at tumor suppressor genes and further alleviate SIRT7 inhibition on tumor suppressor genes. ${ }^{710}$ In addition, SIRT7 targets the oncoprotein MYC to prevent endoplasmic reticulum stress-induced apoptosis, which further promotes cancer cell proliferation and tumor development. ${ }^{11}$ In hepatocellular carcinoma (HCC) cells, SIRT7 increases cell growth by mediating P21 and cyclin D1, which are associated with miR-125a-5p and hypermethylation. ${ }^{12,13}$ Another finding demonstrated that SIRT7 is an integral mediator for cell proliferation via MAPK pathway activation. ${ }^{14}$ Therefore, SIRT7 may play a critical role in colorectal cancer cell proliferation and metastasis. However, the overexpression of SIRT7 may increase cell motility and promote epithelial-mesenchymal transition by decreasing E-cadherin and $\beta$-catenin expression. ${ }^{14}$ In addition, SIRT7 can also regulate DNA repair and maintain genome integrity in several cell lines. ${ }^{15,16}$ Recent studies found that the mRNA of SIRT7 is highly expressed in breast cancer tissue and cell lines, playing a direct role in Akt phosphorylation and modulating cell sensitivity to genotoxic chemotherapeutics in breast cancer cells. ${ }^{17,18}$ However, only a few studies reported the relationship between SIRT7 and long noncoding RNAs (lncRNAs), especially in breast cancer.

lncRNAs are a group of noncoding transcripts longer than 200 nucleotides. LncRNAs may interact with DNA, mRNA, proteins, or other noncoding RNAs and play essential roles in various biological processes. ${ }^{19,20}$ More evidences have suggested that lncRNAs are aberrantly expressed in many cancer types and can be used as cancer biomarkers. LncRNAs are mainly associated with transcription, translation, and chromatin modification in cancer. ${ }^{21}$ Recent studies on breast tumorigenesis have revealed that the lncRNA ANCR regulates the degradation of EZH2 and suppresses breast cancer cell migration and invasion. ${ }^{22}$ In addition, IncRNA CCAT2 promotes breast cancer cell proliferation and tumor formation through the WNT signaling pathway. ${ }^{23}$ LINC00520 is regulated by oncogenic Src, PI3K, and STAT3 and involves in breast cancer cell migration and invasion. ${ }^{24}$ Based on these findings, we speculated that lncRNAs may connect with mRNAs or proteins and play essential roles in breast cancer development.
SIRT7 is highly expressed in breast tissues and cell lines and has essential roles in the malignant progression of breast cancer. ${ }^{17,18,25}$ However, whether the function of SIRT7 in breast cancer is associated with lncRNAs remains unknown. Here, we determine the expression of mRNAs and IncRNAs that are related to SIRT7 using RNA sequencing (RNA-Seq), to obtain a better understanding of the regulatory mechanism of SIRT7 in breast cancer.

\section{Materials and methods Cell culture and siRNA interference}

The cell line MDA-MB-231 was obtained from the Chinese Academy of Sciences Cell Bank (Table S1) and cultured in L-15 medium with $10 \%$ fetal bovine serum (both from Gibco Life Technologies, Carlsbad, CA, USA) under a $37^{\circ} \mathrm{C}$ humidified incubator.

Cells were seeded in a six-well-plate and cultured for 24 hours until they reached to $50 \%-60 \%$ confluency. The cells were transfected with $20 \mu \mathrm{M}$ of either experimental siRNA oligos or nontargeting control with Lipofectamine ${ }^{\circledR}$ 2000 (Invitrogen, Carlsbad, CA, USA). The knockdown efficiency was determined by using qRT-PCR, agarose gel electrophoresis, and Western blot. The sequences of SIRT7 and negative control (NC) siRNAs were synthesized by Shanghai GenePharma (Shanghai, People's Republic of China; Table S2).

\section{Total RNA extraction and qRT-PCR}

The total RNA was extracted by using TRIzol $^{\circledR}$ reagent (Invitrogen) 48 hours after siRNA transfection. Purity and quantity of total RNA were measured by using a NanoDrop ${ }^{\text {TM }}$ equipment (Thermo Fisher Scientific, Waltham, MA, USA). The total RNA was reverse-transcribed using a PrimeScript ${ }^{\mathrm{TM}} \mathrm{RT}$ Master Mix (TaKaRa, Tokyo, Japan). cDNA was quantitated by real-time PCR. The experiments were performed in triplicate on Applied Biosystem's 7500 HT Sequence (Thermo Fisher Scientific). All target genes were normalized to the endogenous reference gene $\beta$-actin by using an optimized comparative $\mathrm{Ct}\left(2^{-\Delta \Delta \mathrm{Ct}}\right)$ value method, where $\Delta \Delta \mathrm{Ct}=\Delta \mathrm{Ct}_{\text {target }}$ $-\Delta \mathrm{Ct}_{\beta \text {-actin }}$. Table $\mathrm{S} 3$ lists the sequences of primers.

\section{Western blot analysis}

Collected cells were placed on ice for 30 minutes to extract proteins into lysis buffer (20 mM Tris, $\mathrm{pH} 7.5,150 \mathrm{mM}$ $\mathrm{NaCl}, 1$ mM EDTA, 1 mM ethylene glycol-bis( $\beta$-aminoethyl ether)-N,N, $\mathrm{N}^{\prime}, \mathrm{N}^{\prime}$-tetraacetic acid, $1 \%$ Triton $\mathrm{X}-100,1 \%$ deoxycholate, $1 \mathrm{mM}$ sodium fluoride, $2 \mathrm{mM}$ sodium 
orthovanadate, and complete protease inhibitor tablets). The lysates were centrifuged at $15,000 \times g$ for 20 minutes at $4^{\circ} \mathrm{C}$, and proteins in the supernatants were quantified using a Bradford assay. Equal amounts of proteins from each sample were mixed with sodium dodecyl sulfate (SDS) sample buffer. Proteins were separated by SDS-polyacrylamide gel electrophoresis and transferred onto a polyvinylidene difluoride membrane. After transferring, the membranes were blocked in Tris-buffered saline containing $0.1 \%$ Tween 20 (TBST) containing 5\% nonfat milk for 1 hour, followed by their incubation at $4{ }^{\circ} \mathrm{C}$ overnight with rabbit monoclonal anti-SIRT7 $(1: 1,000)$ and $\alpha$-tubulin antibody $(1: 2,000)$. After washing thrice in TBST (10 minutes each), the membranes were incubated with secondary antibodies for 1 hour at room temperature. The membranes were processed using an enhanced chemiluminescence detection system (Amersham, Piscataway, NJ, USA). Densitometry was performed by using ImageJ software (National Institutes of Health, Bethesda, MD, USA), and the signal intensity of each protein band was normalized to the respective $\alpha$-tubulin loading control.

\section{Transcriptome assay}

Total RNA with adequate quality was sent to the Beijing Ori-Gene Science and Technology Co., Ltd. for a transcriptome assay. Briefly, the total RNA purity and quantity were measured by using a NanoDrop instrument (Thermo Fisher Scientific). rRNA was removed from total RNA using an Epicenter ribosome kit. After first-strand and second-strand cDNA syntheses, double-stranded cDNA was purified using $1.8 \times$ Agencourt AMPure XP beads. Following fragment screening, library building, and PCR product purification, the samples were sequenced in an Illumina HiSeq ${ }^{\circledR} 2500$ (Illumina, San Diego, CA, USA). This array contains 13,179 lncRNA probes and 19,831 coding transcript probes, and these were constructed through the most authoritative public transcriptome database, Gencode. Data are accessible at the NCBI SRA database (accession number SRP107736).

\section{Gene ontology (GO) and pathway analyses}

GO and pathway analyses were used to determine the potential roles of differentially expressed lncRNAs and mRNAs in GO terms or biological pathways. GO analysis (www.geneontology.org) was used to investigate biological functions. This analysis classifies the functions of these differentially expressed genes according to the following three aspects: biological process, cellular component, and molecular function. Fisher's exact test was applied to classify the $\mathrm{GO}$ category. The $p$-value denotes the significance of $\mathrm{GO}$ term enrichment in the dysregulated genes. The lower the $p$-value, the more significant is the GO term $(p<0.05$ was selected as a threshold).

Pathway analysis was used to investigate the differentially expressed coding genes according to the Kyoto Encyclopedia of Genes and Genomes (KEGG) (http://www.genome.jp/ kegg/). The $p$-value indicates the significance of the pathway correlated with the conditions. A value of $p<0.05$ was considered statistically significant.

\section{LncRNA-mRNA coexpression network}

LncRNA-mRNA coexpression network was constructed based on the correlation between the differentially expressed lncRNAs and mRNAs. We selected lncRNAs from the most significant profiles to conduct the lncRNA-mRNA coexpression network. In this network, blue represents downregulation, red represents upregulation, circle represents mRNA, square represents lncRNA, and the lines between cycle nodes represent interactions between lncRNA and mRNA. The degree is defined as the number of one lncRNA directly to the mRNAs. ${ }^{26}$ The higher the degree, the more central is the lncRNA or mRNA in the coexpression network.

\section{Statistical analysis}

All data are presented as means \pm standard error of mean. All experimental assays were performed in triplicates. Statistical comparisons were made by using the analysis of variance or independent $t$-test, followed by Duncan's multiple comparisons test. A $p$-value of $<0.05$ was considered significant.

\section{Results \\ Efficiency of SIRT7 knockdown in MDA-MB-23 I cells}

The mRNA expression level of SIRT7 was examined by qRT-PCR and agarose gel electrophoresis after the transfection of MDA-MB-231 cells with three pairs of SIRT7 siRNA. As shown in Figure 1A and B, the mRNA level of SIRT7 was significantly lower in cells from all three treatment groups as compared to cells in the NC group $(p<0.05)$, and the best knockdown efficiency was obtained with siSIRT7-2. Moreover, the protein levels of SIRT7 were also significantly reduced after SIRT7 siRNA transfection, as shown in Figure 1C and D. Hence, we chose siSIRT7-2 for the subsequent experiment. 


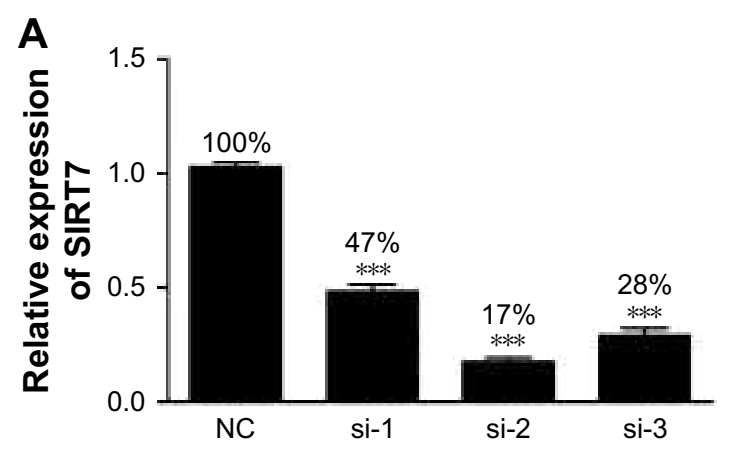

B

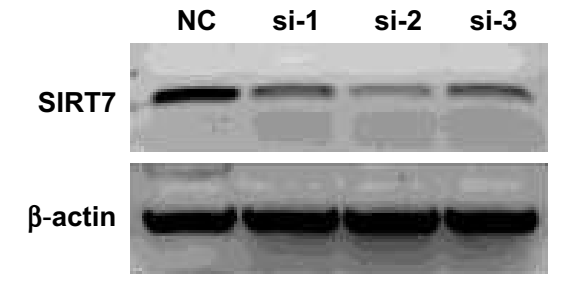

C
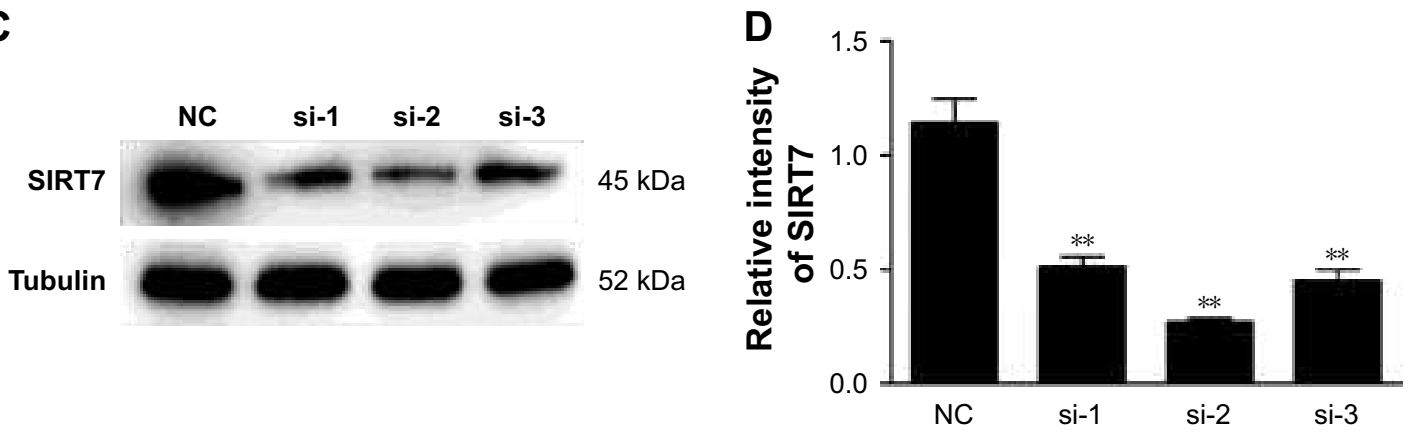

Figure I The mRNA expression and protein levels were detected by qRT-PCR (A), agarose gel electrophoresis (B), and Western blot (C, D) after transfection of MDA-MB-23I breast cancer cells with three different pairs of siRNA. si-I, si-2, and si-3 represent different sequences of siSIRT7. $* * p<0.0$ I and $* * * p<0.00$ I.

Abbreviation: NC, negative control.

\section{Differentially expressed mRNAs and IncRNAs in MDA-MB-23 I cells treated with NC and siSIRT7}

We investigated the differential expression levels of mRNAs and lncRNAs in MDA-MB-231 cells treated with siSIRT7 using RNA-Seq. As shown in Figure 2A and B, hierarchical clustering revealed the differential expressions of mRNAs and IncRNAs. After filtering, a total of 240 differentially expressed mRNAs, including 87 downregulated genes and 153 upregulated genes, were identified between siSIRT7treated and NC-treated cells (Figure 2C; $<<0.05$ ), and Table 1 lists the top 20 differential expressions of mRNAs $(p<0.05)$. The expression profiling data suggested the differentially expressed 26 lncRNAs between the two groups (Table 2; $p<0.05$ ), including 23 downregulated genes and three upregulated genes (Figure 2D; $p<0.05$ ).

To verify the reliability of RNA-Seq results, a qRT-PCR assay was performed to detect the expression levels of five randomly selected mRNAs and four lncRNAs from the two groups. Table S2 lists all the primers used in this study. In agreement with the RNA-Seq results, the mRNA levels of ATF2, CCNG2, E2F2, RCHY1, and HSPB1 were significantly different between the two groups (Figure 3A). The expression levels of IncRNA RP11-160E2.6, LIN01002, LNC00630, and LIPE-AS1 were consistent with those observed in RNA-Seq (Figure 3B). We used another breast cancer cell line MCF-7 to further validate these results and observed that the expression trends of these mRNAs and lncRNAs in MCF-7 cells after SIRT7 depletion were consistent with those observed in MDA-MB-231 cells (Figure 3C and D).

\section{GO and KEGG pathway analyses of differentially expressed mRNAs}

We thoroughly explored the potential functions and mechanisms of these differentially expressed mRNAs by performing GO analysis of SIRT7 knockdown in MDA-MB-231 cells. GO analysis results indicate that these genes were enriched in biological processes that are associated with cellular processes, biological regulation, metabolic process, and signal-organism process (Figure 4A). The cellular component of these genes included cell, cell part, membrane, and organelle (Figure 4B); the molecular functions of these genes were binding, catalytic activity, transporter activity, and molecular transducer activity (Figure 4C). In addition, we found that the highest $\mathrm{GO}$ enrichments of the upregulated differentially expressed mRNAs were DNA replication (GO, biological processes), nucleoplasm (GO, cellular components), and CXCR chemokine receptor binding (GO, molecular function) as shown in Figure 5A-C. However, the highest GO enrichments of the downregulated differentially expressed mRNAs were maturation of large subunit rRNA from 

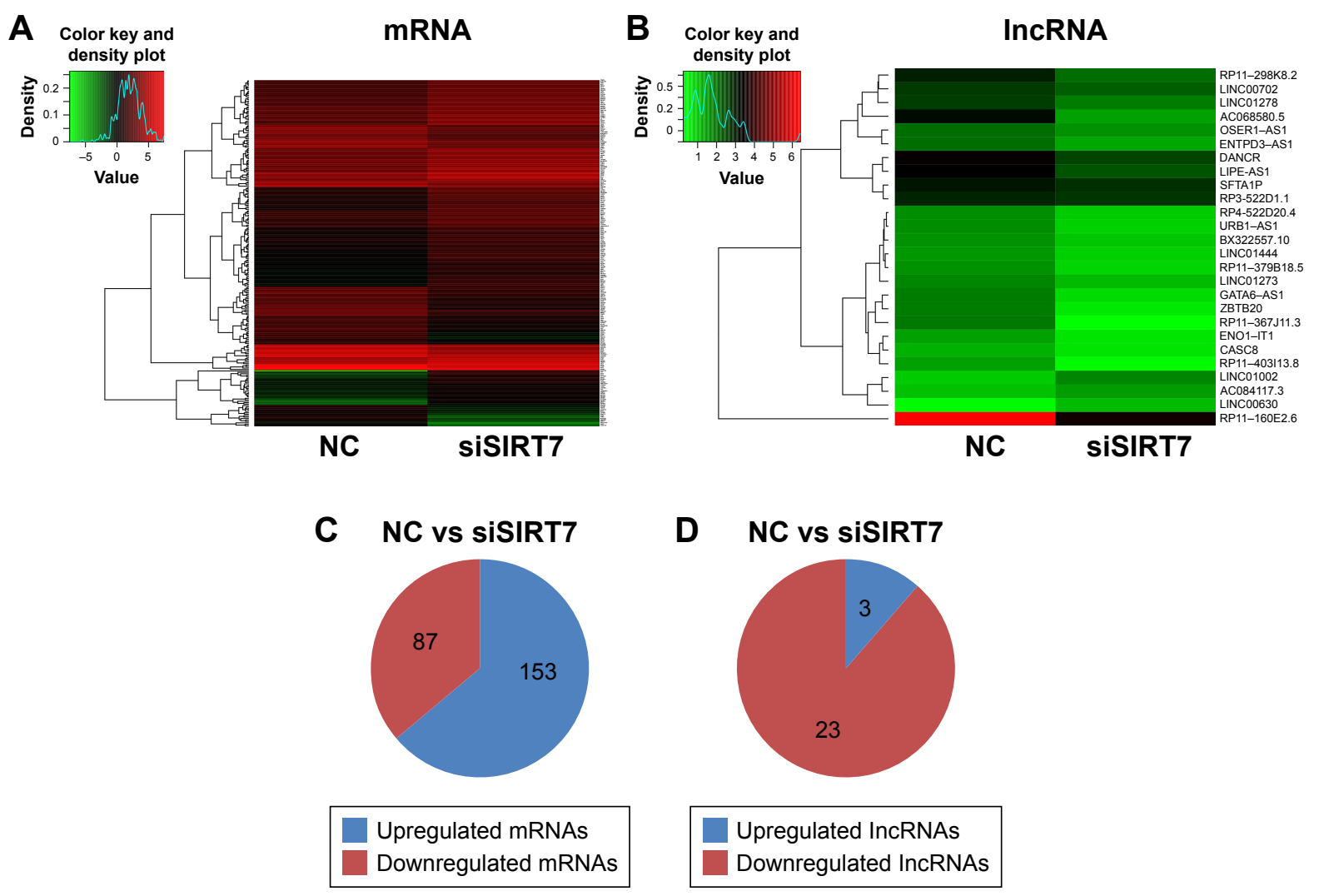

Figure 2 Differentially expressed mRNAs (A) and IncRNAs (B) were analyzed by hierarchical clustering within NC and siSIRT7 groups in MDA-MB-23I cells; red indicates high relative expression, and green indicates low relative expression. (C, D) The number of mRNAs and IncRNAs differentially expressed. Abbreviation: NC, negative control.

tricistronic rRNA transcript (GO, biological processes), mitochondrion (GO, cellular components), and $\mathrm{NAD}^{+}$ ADP-ribosyltransferase activity (GO, molecular function; Figure 6A-C).

Table I Top 20 differentially expressed of mRNAs

\begin{tabular}{lll}
\hline Gene ID & mRNA & Style \\
\hline ENSG00000122642 & FKBP9 & Down \\
ENSG0000016396I & RNFI68 & Down \\
ENSG00000166073 & GPRI76 & Down \\
ENSG00000265972 & TXNIP & Down \\
ENSG00000246705 & H2AFJ & Down \\
ENSG00000177034 & MTX3 & Down \\
ENSG00000090530 & P3H2 & Down \\
ENSG00000101I52 & DNAJC5 & Down \\
ENSG00000119408 & NEK6 & Down \\
ENSG00000130303 & BST2 & Down \\
ENSG00000170542 & SERPINB9 & Up \\
ENSG00000180694 & TMEM64 & Up \\
ENSG00000152332 & UHMKI & Up \\
ENSG00000128918 & ALDHIA2 & Up \\
ENSG00000131023 & LATSI & Up \\
ENSG00000131473 & ACLY & Up \\
ENSG00000138160 & KIFII & Up \\
ENSG00000162607 & USPI & Up \\
ENSG00000165244 & ZNF367 & Up \\
ENSG00000163739 & CXCLI & Up \\
\hline
\end{tabular}

Table 2 Differentially expressed IncRNAs

\begin{tabular}{lll}
\hline Gene ID & InCRNA & Style \\
\hline ENSG00000245468 & RPII-367III.3 & Down \\
ENSG00000262202 & RPII-I60E2.6 & Down \\
ENSG000002724I9 & RPII-403II3.8 & Down \\
ENSG000002660I0 & GATA6-ASI & Down \\
ENSG00000236269 & ENOI-ITI & Down \\
ENSG00000226950 & DANCR & Down \\
ENSG00000I8I722 & ZBTB20 & Down \\
ENSG000002295I2 & AC068580.5 & Down \\
ENSG00000256073 & URBI-ASI & Down \\
ENSG0000024I288 & RPII-379BI8.5 & Down \\
ENSG000002I3904 & LIPE-ASI & Down \\
ENSG00000272I2I & RP4-555D20.4 & Down \\
ENSG00000224985 & RPII-297K8.2 & Down \\
ENSG0000026430I & LINC0I444 & Down \\
ENSG00000235437 & LINC0I278 & Down \\
ENSG00000223797 & ENTPD3-ASI & Down \\
ENSG00000225383 & SFTAIP & Down \\
ENSG00000246228 & CASC8 & Down \\
ENSG0000023I742 & LINC0I273 & Down \\
ENSG00000233II7 & LINC00702 & Down \\
ENSG00000224I67 & RP3-522DI.I & Down \\
ENSG0000022389I & OSERI-ASI & Down \\
ENSG000002I5447 & BX322557.I0 & Down \\
ENSG00000256006 & AC084II7.3 & Up \\
ENSG00000282508 & LINC0I002 & Up \\
ENSG00000223546 & LINC00630 & Up \\
\hline
\end{tabular}


A

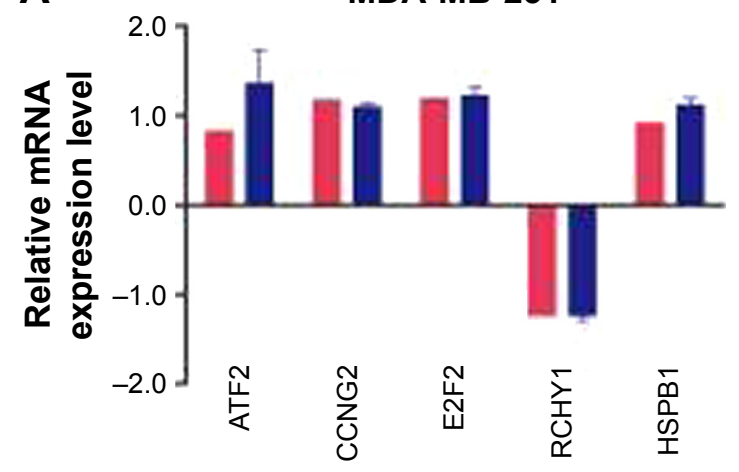

B

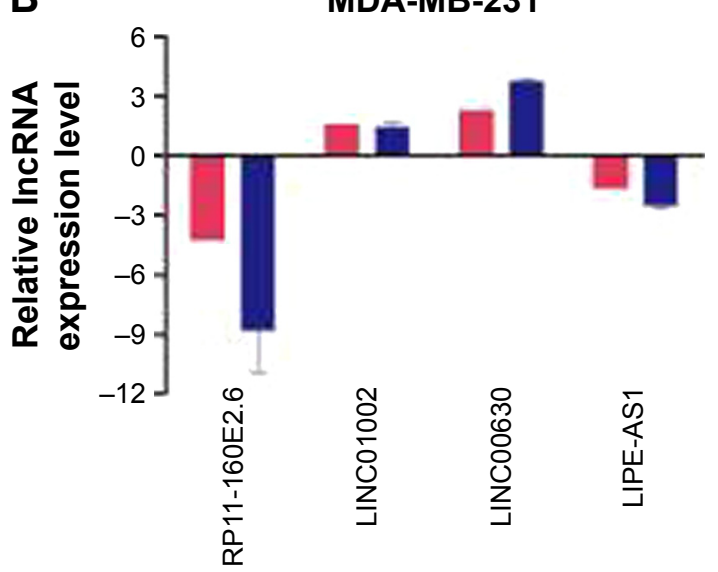

RNA-Seq $\square$ qRT-PCR

C

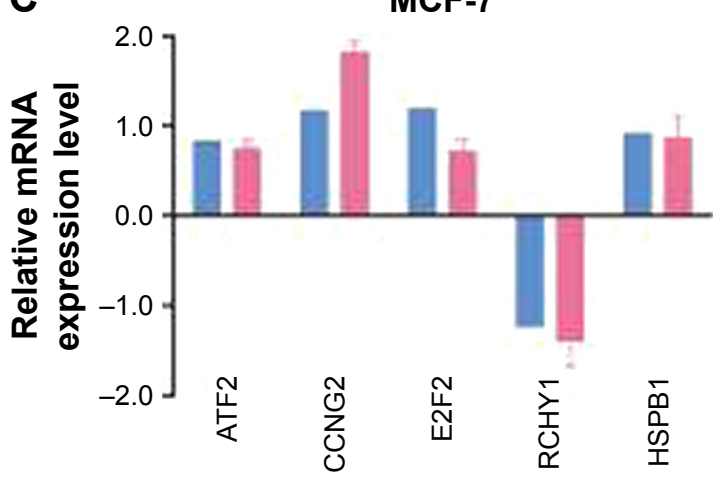

D

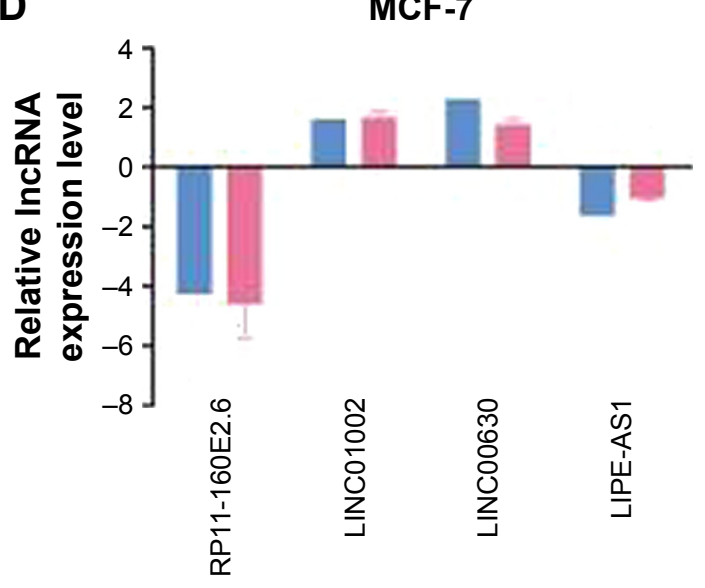

RNA-Seq $\equiv$ qRT-PCR

Figure 3 qRT-PCR was performed to confirm the validity of the RNA-Seq data in MDA-MB-23I and MCF-7 cells. The mRNAs (A, C) and IncRNAs (B, D) were expressed as log2 values (RNA-Seq) and $-\Delta \Delta C t$ values (qRT-PCR).

Abbreviation: RNA-Seq, RNA sequencing.

KEGG pathway analysis was employed to examine the biological pathways of the differentially expressed mRNAs. The results indicated that these genes were mainly involved in MAPK signaling pathway; cytokine-cytokine receptor interaction; and cancer-related, cell cycle, and tumor necrosis factor (TNF) signaling pathways (Figure 7A). In addition, there were 22 upregulated and four downregulated KEGG pathways $(p<0.05)$. Figure $7 \mathrm{~B}$ and $\mathrm{C}$ shows the most upregulated and downregulated pathways.

\section{GO and KEGG pathway analyses of the target genes of the differentially expressed IncRNAs}

To further explore the functions of the differentially expressed lncRNAs, we performed GO and KEGG pathway analyses for the target mRNAs of these lncRNAs. Among these target mRNAs, 108 mRNAs were assigned to biological process; 15 mRNAs were assigned to cellular component and 32 mRNAs to molecular process. The significant enrichment of each GO term was evaluated at $p<0.05$. In subsequent steps, the GO terms of biological process were filtered by the enrichment scores $(-\operatorname{Lg}(\mathrm{P}))$. Figure 8A presents the top $10 \mathrm{GO}$ terms. The results showed that the carboxylic acid metabolic process was the most enriched GO terms targeted in biological process.

We performed KEGG pathway analysis to explore the biological pathways for the target genes of the differentially expressed lncRNAs. Our results showed that these mRNAs were involved in seven biological pathways. The top three enriched pathways were glycolysis, propanoate metabolism, 
A Biological process

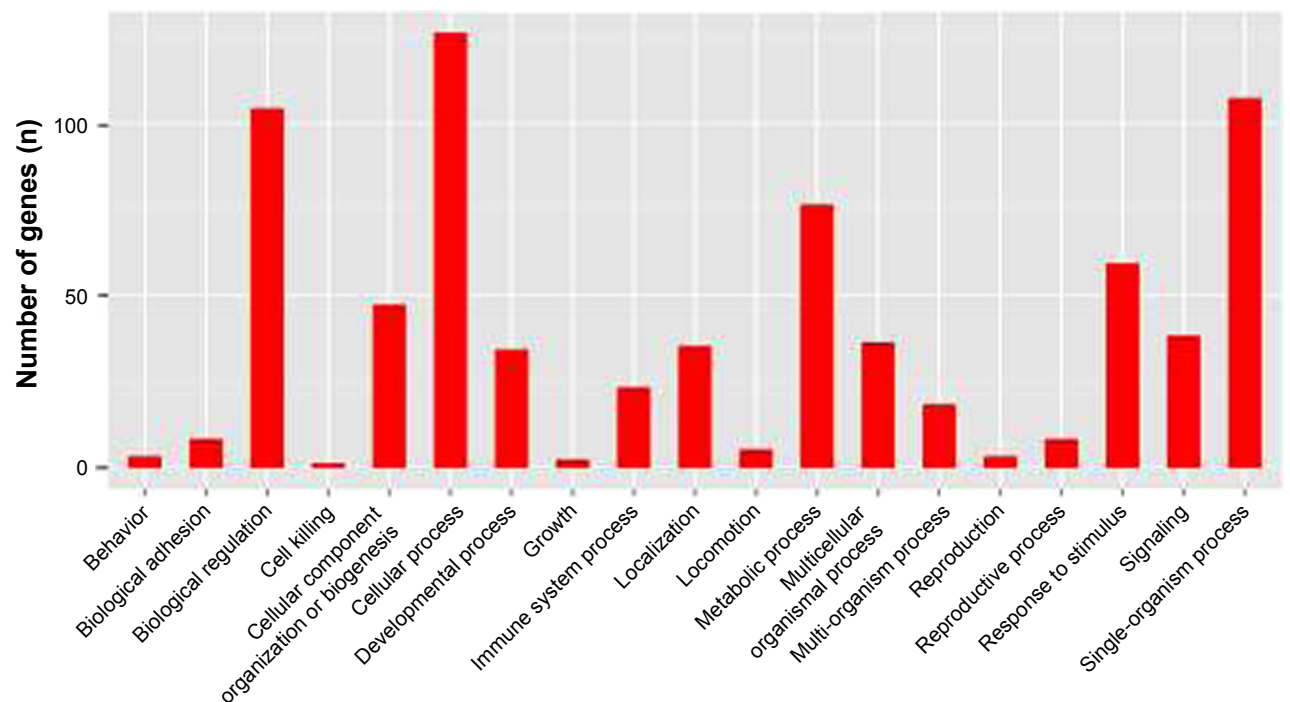

B

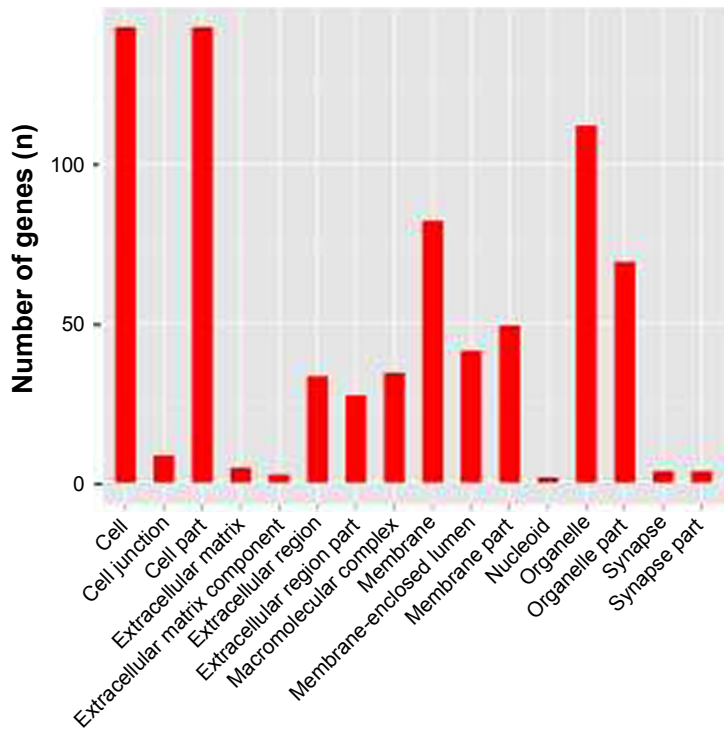

C

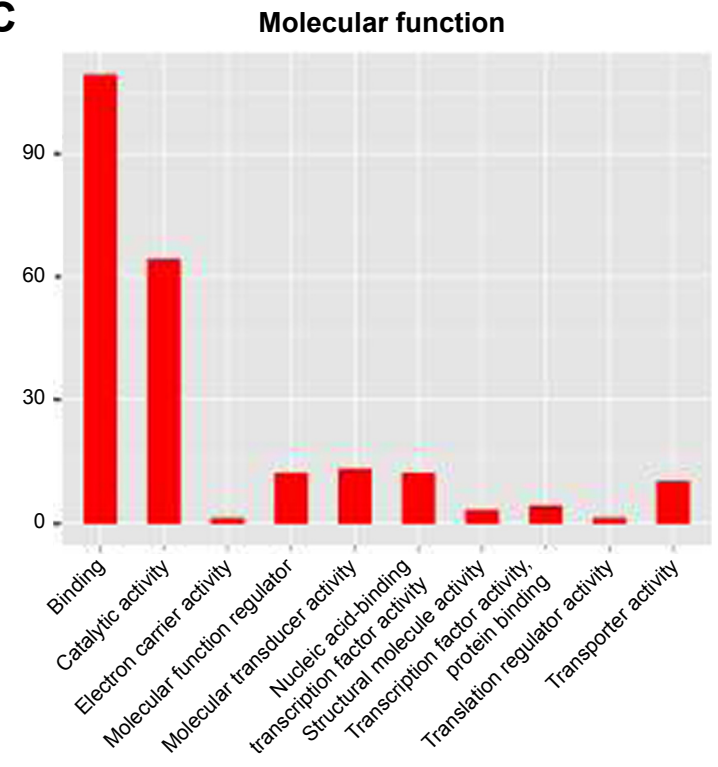

Figure $4 \mathrm{GO}$ analysis of the differentially expressed genes after depletion of SIRT7. (A) GO analysis results showed that these mRNAs were enriched in the biological functions related to cellular processes, biological regulation, metabolic process, and signal-organism process. (B) The cellular component of these genes included cell, cell part, membrane, and organelle. (C) The molecular function of these genes composed of binding, catalytic activity, transporter activity, and molecular transducer activity. Abbreviation: GO, gene ontology.

and cysteine and methionine metabolism. Figure 8B shows the enrichment analysis of these pathways.

\section{LncRNA-mRNA coexpression network}

Recent studies have demonstrated that lncRNAs are essential for gene transcription. ${ }^{27,28}$ Therefore, to further examine the function of these differentially expressed lncRNAs in breast cancer cells, we constructed the lncRNA-mRNA coexpression network between 240 differentially expressed mRNAs and 26 differentially expressed lncRNAs. Our results showed that 186 mRNAs and 23 lncRNAs were included in the coexpression network, wherein box nodes represent lncRNAs and circular nodes represent mRNAs (Figure 9). The value of "degree" in the coexpression network suggests that one mRNA/lncRNA may correlate with several mRNAs/lncRNAs. The results showed that IncRNA GATA6-AS1, KINC01002, LIPE-AS1, RP11403I13.8, BX322557.10, RP11-367J11.3, RP11-160E2.6, ZBTB20, AC068580.5, and RP11-379B18.5 exhibited a high degree of connectivity and may play key roles in this network (Table 3). Above all, our results highlight the potential internal adjustment correlation between the 


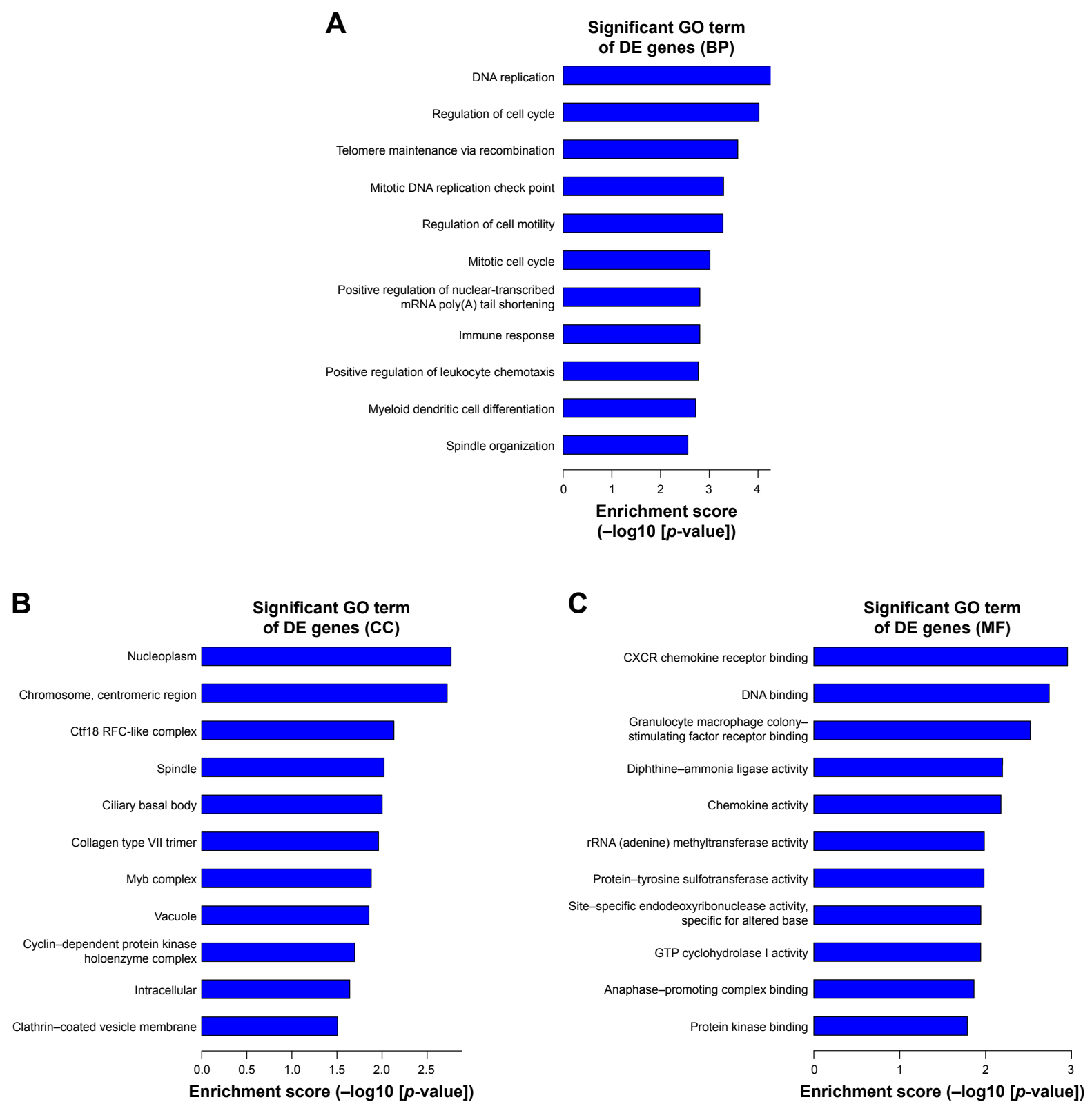

Figure 5 The top $10 \mathrm{GO}$ enrichment analysis of the upregulated mRNAs: (A) biological process, (B) cell component, and (C) molecular function. Abbreviations: DE, differentially expressed; GO, gene ontology.

differentially expressed lncRNAs and mRNAs that are regulated by SIRT7 in breast cancer.

\section{Discussion}

Breast cancer is the most common cancer diagnosed in women and the second leading cause of cancer-related death among women around the world. ${ }^{29}$ The occurrence of breast cancer is a complex process caused by multiple genetic, epigenetic, and environmental factors. ${ }^{30}$ The sirtuin family is reported to play wide roles associated with metabolism, cancer, transcriptional silencing, chromosomal stability, stress response, cell differentiation, inflammation, apoptosis, and DNA repair. ${ }^{31,32}$ SIRT7 is a member of the sirtuins family that plays critical roles in cancer development, but based on the previous studies, SIRT7 has an opposite expression trend in different cancers and also plays different functions in different kinds of tumor development. ${ }^{33}$ For example, knockdown of SIRT7 inhibits the proliferation of U2OS cells, induces apoptosis, and also reduces the development of human U251 cancer cell xenografts in mice, ${ }^{7,34}$ but SIRT7 is negatively correlated with the progression of human head and neck squamous cell carcinoma. ${ }^{35}$ In the meantime, the potential mechanism of SIRT7 in cancer development was still not well clarified, especially in breast cancer. 


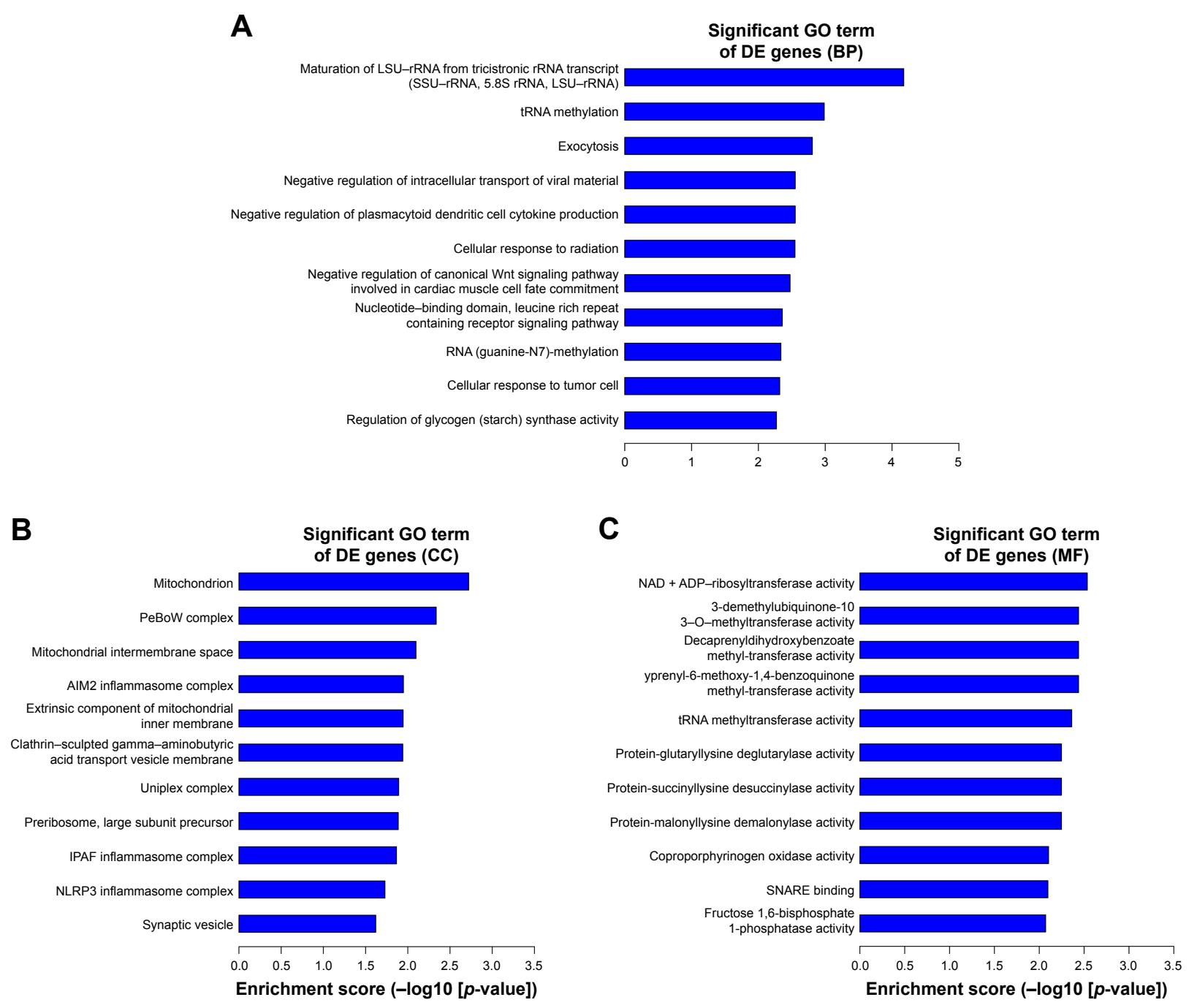

Figure 6 The top $10 \mathrm{GO}$ enrichment analysis of the downregulated mRNAs: (A) biological process, (B) cell component, and (C) molecular function. Abbreviations: DE, differentially expressed; GO, gene ontology.

LncRNAs are involved in almost each level of gene expression and have diverse functions, such as genome rearrangement, chromatin modification, imprinting, transcription, splicing, and translation. ${ }^{36-38}$ Recent studies showed that IncRNAs played important roles in pathogenesis and tumorigenesis and could be novel biomarkers and potential therapeutic targets in diseases. LncRNAs are aberrantly expressed in various types of cancer, including glioma, colorectal, hepatocellular, and breast cancers. ${ }^{39-42}$ The role of lncRNAs as drivers of tumor suppressive functions has been highlighted in diverse cancer types. For example, lncRNA RP11-62F24.2 was significantly upregulated in gastric cancer tissues and correlated with invasion and tumor size, ${ }^{43}$ and lncRNA PURPL promotes tumorigenicity in colorectal cancer by suppressing basal p53 levels. ${ }^{44}$ In contrast, the function of SIRT7 regulates the expression of IncRNA in breast cancer has not been reported. In the present study, we performed RNA-Seq to determine the differential expressions of mRNAs and lncRNAs after knockdown of SIRT7 in MDA-MB-231 cells. According to RNA-Seq outcomes, we identified 240 differentially expressed mRNAs and 26 differentially expressed lncRNAs in SIRT7-depleted MDA-MB-231 breast cancer cells. To further illuminate the potential biological functions of the differentially expressed genes, we first performed GO and KEGG pathway analyses to predict the biological functions of these differentially expressed mRNAs after SIRT7 depletion. We mainly enriched the mRNAs that regulated biological processes, and the top three enrichment terms of upregulated mRNAs were regulation of DNA replication, cell cycle regulation, and telomere maintenance via recombination. Therefore, it is reasonable to infer that SIRT7 may mainly regulate DNA replication, cell cycle, and senescence, which in turn affect breast cancer cell development. 


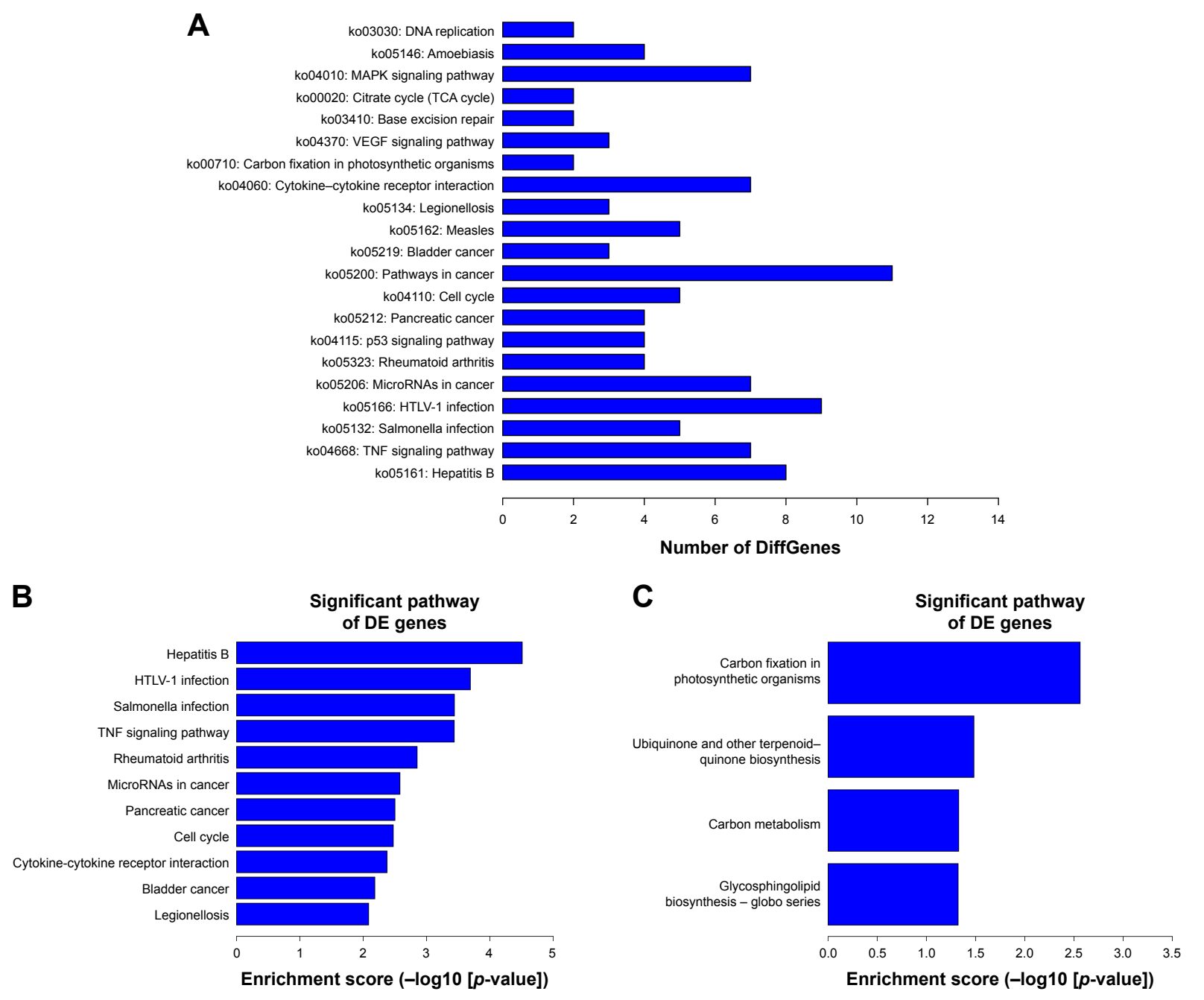

Figure 7 KEGG analysis of the differentially expressed mRNAs. (A) The KEGG analysis results showed that the most enriched pathways included MAPK signaling pathway; those involved in cancer, HTLV-I, and hepatitis B infection; and TNF signaling pathway. (B, C) The top 10 pathway corresponding to the upregulated mRNAs and downregulated mRNAs.

Abbreviations: DE, differentially expressed; HTLV-I, human T-cell lymphotropic virus type I; KEGG, Kyoto Encyclopedia of Genes and Genomes; TCA, tricarboxylic acid; TNF, tumor necrosis factor.

A

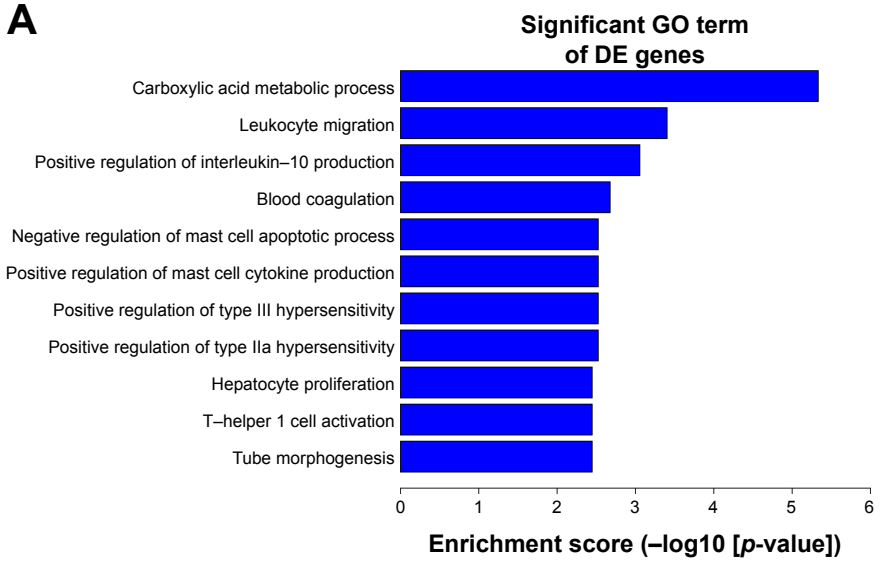

B

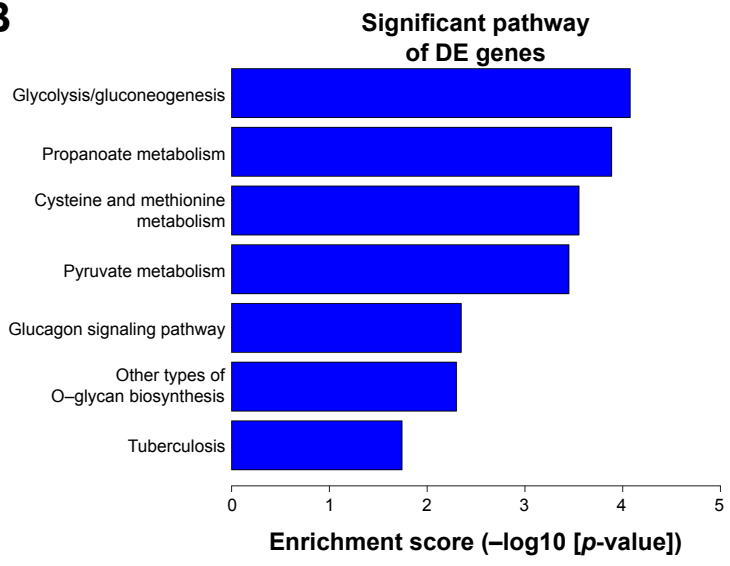

Figure $8 \mathrm{GO}$ and KEGG analyses of the target genes of these differentially expressed IncRNAs. (A) Top $10 \mathrm{GO}$ terms of biological processes for IncRNA-targeted genes. (B) Top 10 pathways corresponding to the IncRNA-targeted genes.

Abbreviations: DE, differentially expressed; GO, gene ontology; KEGG, Kyoto Encyclopedia of Genes and Genomes. 


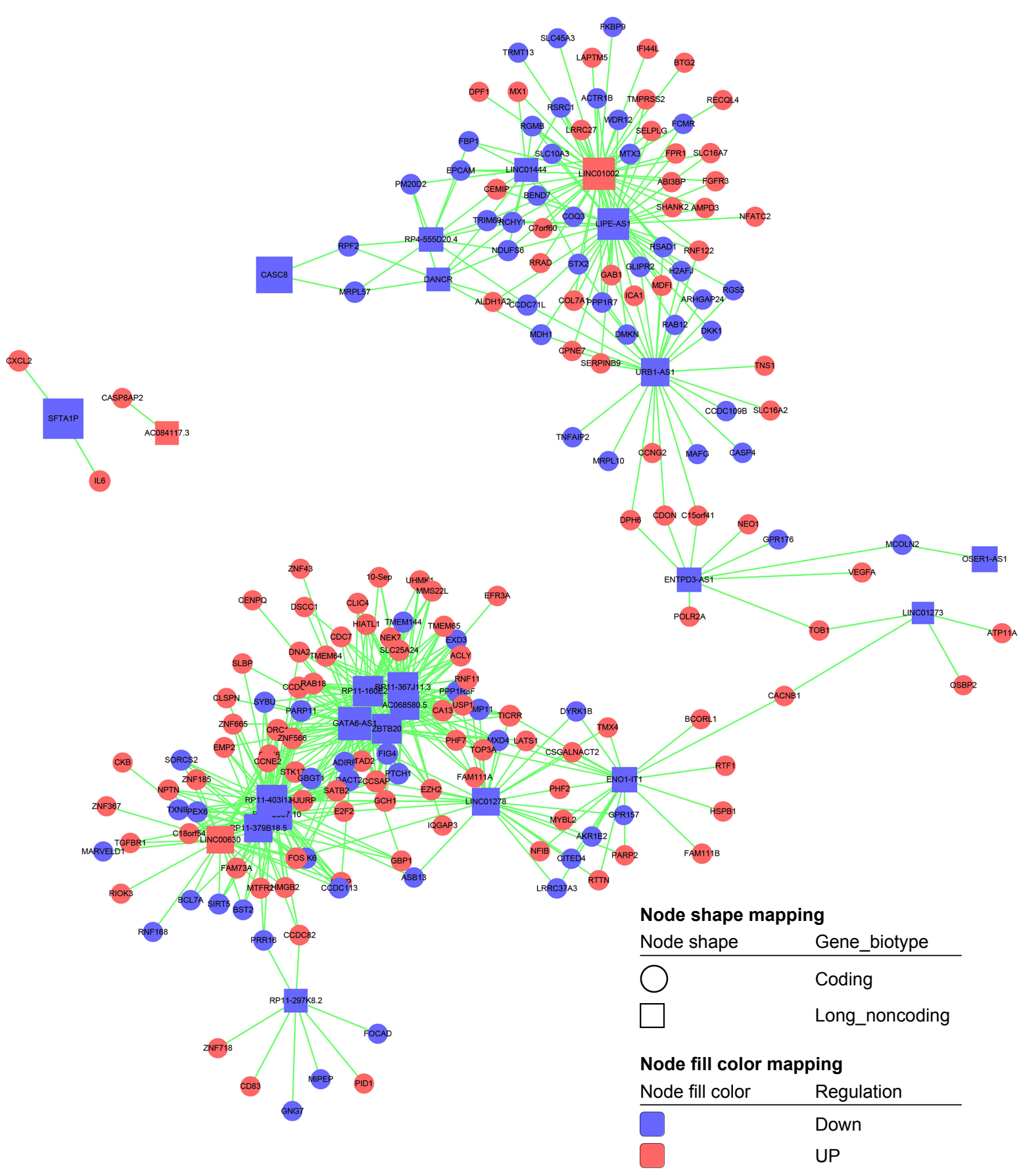

Figure 9 The differentially expressed mRNA-IncRNA coexpression network. Box nodes represent IncRNAs, while circular nodes represent mRNAs. Red represents upregulation, and blue represents downregulation.

For instance, a recent study showed that SIRT7 promotes cellular survival under conditions of genomic stress through attenuating DNA damage and activation of stressactivated protein kinase. ${ }^{45}$ Knockdown of SIRT7 may inhibit cell proliferation, induce cell apoptosis, and reduce tumor growth through alterations in cyclin D1 and p21 expression. ${ }^{12,46}$ In aged human stem cells, the expression of SIRT7 was decreased, and the overexpression of SIRT7 may attenuate doxorubicin-induced premature senescence. ${ }^{47,48}$
Taken together, these data suggest an alternative mechanism for SIRT7, which necessitates further studies. Furthermore, pathway analysis showed that these differentially expressed mRNAs were mainly involved in MAPK signaling pathway; pathways in cancer, hepatitis B, and human T-cell lymphotropic virus type I infection; cytokinecytokine receptor interaction; and TNF signaling pathway. The most enriched pathway was that involved in hepatitis B infection. A significant exposure to hepatitis B virus 
Table 3 The "degree" of IncRNAs in coexpression network $\left(<30^{\circ}\right.$ was not shown)

\begin{tabular}{llll}
\hline LncRNA & Type & Style & Degree \\
\hline GATA6-ASI & Noncoding & Down & 49 \\
LINC0I002 & Noncoding & UP & 45 \\
LIPE-ASI & Noncoding & Down & 43 \\
RPII-403II3.8 & Noncoding & Down & 40 \\
BX322557.I0 & Noncoding & Down & 39 \\
RPII-367III.3 & Noncoding & Down & 38 \\
RPII-I60E2.6 & Noncoding & Down & 36 \\
ZBTB20 & Noncoding & Down & 34 \\
AC068580.5 & Noncoding & Down & $3 I$ \\
RPII-379BI8.5 & Noncoding & Down & 30 \\
\hline
\end{tabular}

infection is a carcinogenic factor, which may lead to the early development of breast cancer. ${ }^{49}$ Therefore, SIRT7 plays essential roles in hepatitis B virus infection and indirectly regulates breast cancer development; further studies are needed to elucidate this mechanism.

Although previous studies have reported the involvement of SIRT7 in the development of several types of cancers, the function of SIRT7 in breast cancer and its association with $\operatorname{lncRNAs}$ remain unknown. RNA-Seq analysis revealed that 26 lncRNAs showed significant differential expressions after SIRT7 depletion, indicating the association between SIRT7 and these lncRNAs during breast cancer development. It is well known that lncRNAs regulate the neighboring protein-coding genes. ${ }^{50}$ To further explore the possible functional roles of these lncRNAs in breast cancer, we examined the target genes of these differentially expressed lncRNAs by GO and pathway analyses. GO analysis revealed that these lncRNAs were most enriched in carboxylic acid metabolic process, which is closely associated with cancer development. Moreover, pathway analysis suggested the involvement of these lncRNAs in glycolysis, propanoate metabolism, and cysteine and methionine metabolism, indicative of their participation in metabolic processes. Depleting of lncRNA ceruloplasmin resulted in a significant increase in apoptosis and decreased glycolysis and proliferation in cancer cells via mediating the expression of glucoase-6-phosphate isomerase. ${ }^{51}$ Therefore, SIRT7 may also be involved in breast cancer cell proliferation by glycolysis. Above all, SIRT7 may play a critical role in metabolic processes through these differentially expressed lncRNAs in breast cancer development.

Based on the differentially expressed mRNAs and lncRNAs, we developed a coexpression network to detect key mRNAs and lncRNAs related to SIRT7 in breast cancer development. LncRNA GATA6-AS1, KINC01002, LIPE-AS1, RP11-403I13.8, BX322557.10, RP11-367J11.3, RP11-160E2.6, ZBTB20, AC068580.5, and RP11-379B18.5 were regulated by
SIRT7 and exhibited highest degree of connectivity, suggestive of their critical roles in the coexpression network. For instance, ZBTB20 gene is a new member of the BTB/POZ-ZF protein complex that is highly expressed in HCC and associated with poor prognosis in patients with HCC. ${ }^{52}$ However, to date, very few reports have explored the relationship between lncRNAs and SIRT7. Our results indicate that these IncRNAs may affect breast cancer cell proliferation and development by influencing the expression of their neighboring mRNAs, which were regulated by SIRT7. However, the analysis of the direct relationship between lncRNAs and SIRT7 in breast cancer requires further investigation and will be valuable for clinical diagnosis.

\section{Conclusion}

The present study using RNA-Seq data offers newfound information about the potential regulatory mechanism of SIRT7 in breast cancer. Our results indicate that the function of SIRT7 in breast cancer development may be mediated through various signaling pathways, including the MAPK signaling pathway, cytokine-cytokine receptor interaction, and pathways in cancers and cell cycle, and TNF signaling pathway. In addition, SIRT7 may regulate breast cancer cell proliferation and tumor development through lncRNAs GATA6-AS1, KINC01002, LIPE-AS1, RP11-403I13.8, BX322557.10, RP11-367J11.3, RP11-160E2.6, ZBTB20, AC068580.5, and RP11-379B18.5. Thus, our study provides valuable insights for future research, which may be directed to confirm the exact relationship between SIRT7 and these lncRNAs and the function of these lncRNAs in breast cancer.

\section{Acknowledgments}

This work was supported by the National Natural Science Foundation of China (Grant Nos 31772567 and 31372290) and the Fundamental Research Funds for the Central Universities (Grant No KJQN201607).

\section{Disclosure}

The authors report no conflicts of interest in this work.

\section{References}

1. Haigis MC, Sinclair DA. Mammalian sirtuins: biological insights and disease relevance. Annu Rev Pathol. 2010;5:253-295.

2. Imai S, Guarente L. NAD+ and sirtuins in aging and disease. Trends Cell Biol. 2014;24(8):464-471.

3. Nakagawa T, Guarente L. Sirtuins at a glance. J Cell Sci. 2011;124(Pt 6): 833-838.

4. Roth M, Chen WY. Sorting out functions of sirtuins in cancer. Oncogene. 2014;33(13):1609-1620.

5. Bosch-Presegue L, Vaquero A. The dual role of sirtuins in cancer. Genes Cancer. 2011;2(6):648-662.

6. Michishita E, Park JY, Burneskis JM, Barrett JC, Horikawa I. Evolutionarily conserved and nonconserved cellular localizations and functions of human SIRT proteins. Mol Biol Cell. 2005;16(10):4623-4635. 
7. Barber MF, Michishita-Kioi E, Xi Y, et al. SIRT7 links H3K18 deacetylation to maintenance of oncogenic transformation. Nature. 2012; 487(7405):114-118.

8. Horwitz GA, Zhang K, McBrian MA, Grunstein M, Kurdistani SK, Berk AJ. Adenovirus small ela alters global patterns of histone modification. Science. 2008;321(5892):1084-1085.

9. Seligson DB, Horvath S, McBrian MA, et al. Global levels of histone modifications predict prognosis in different cancers. Am J Pathol. 2009; 174(5):1619-1628.

10. Lee HS, Jung W, Lee E, et al. SIRT7, H3K18ac, and ELK4 immunohistochemical expression in hepatocellular carcinoma. J Pathol Transl Med. 2016;50(5):337-344.

11. Silvera D, Formenti SC, Schneider RJ. Translational control in cancer. Nat Rev Cancer. 2010;10(4):254-266.

12. Kim JK, Noh JH, Jung KH, et al. Sirtuin7 oncogenic potential in human hepatocellular carcinoma and its regulation by the tumor suppressors MiR-125a-5p and MiR-125b. Hepatology. 2013;57(3):1055-1067.

13. Han Y, Liu Y, Zhang H, et al. Hsa-miR-125b suppresses bladder cancer development by down-regulating oncogene SIRT7 and oncogenic long non-coding RNA MALAT1. FEBS Lett. 2013;587(23): 3875-3882.

14. Yu H, Ye W, Wu J, et al. Overexpression of sirt7 exhibits oncogenic property and serves as a prognostic factor in colorectal cancer. Clin Cancer Res. 2014;20(13):3434-3445.

15. Li L, Shi L, Yang S, et al. SIRT7 is a histone desuccinylase that functionally links to chromatin compaction and genome stability. Nat Commun. 2016;7:12235.

16. Vazquez BN, Thackray JK, Simonet NG, et al. SIRT7 promotes genome integrity and modulates non-homologous end joining DNA repair. EMBO J. 2016;35(14):1488-1503.

17. Geng Q, Peng H, Chen F, Luo R, Li R. High expression of Sirt7 served as a predictor of adverse outcome in breast cancer. Int J Clin Exp Pathol. 2015;8(2):1938-1945.

18. Aljada A, Saleh AM, Alkathiri M, Shamsa HB, Al-Bawab A, Nasr A. Altered sirtuin 7 expression is associated with early stage breast cancer. Breast Cancer (Auckl). 2015;9:3-8.

19. Li X, Wu Z, Fu X, Han W. IncRNAs: insights into their function and mechanics in underlying disorders. Mutat Res Rev Mutat Res. 2014 762:1-21.

20. Wang KC, Chang HY. Molecular mechanisms of long noncoding RNAs. Mol Cell. 2011;43(6):904-914.

21. Lee JT. Epigenetic regulation by long noncoding RNAs. Science. 2012;338(6113):1435-1439.

22. Li Z, Hou P, Fan D, et al. The degradation of EZH2 mediated by lncRNA ANCR attenuated the invasion and metastasis of breast cancer Cell Death Differ. 2017;24:59-71.

23. Cai Y, He J, Zhang D. Long noncoding RNA CCAT2 promotes breast tumor growth by regulating the Wnt signaling pathway. Onco Targets Ther. 2015;8:2657-2664.

24. Henry WS, Hendrickson DG, Beca F, et al. LINC00520 is induced by Src, STAT3, and PI3K and plays a functional role in breast cancer. Oncotarget. 2016;7:81981-81994.

25. Ashraf N, Zino S, Macintyre A, et al. Altered sirtuin expression is associated with node-positive breast cancer. Br J Cancer. 2006;95(8): 1056-1061.

26. Barabasi AL, Oltvai ZN. Network biology: understanding the cell's functional organization. Nat Rev Genet. 2004;5(2):101-113.

27. Sun Y, Wei G, Luo H, et al. The long noncoding RNA SNHG1 promotes tumor growth through regulating transcription of both local and distal genes. Oncogene. Epub 2017 Aug 21.

28. Touat-Todeschini L, Shichino Y, Dangin M, et al. Selective termination of lncRNA transcription promotes heterochromatin silencing and cell differentiation. EMBO J. 2017;36(17):2626-2641

29. DeSantis CE, Bray F, Ferlay J, Lortet-Tieulent J, Anderson BO, Jemal A. International variation in female breast cancer incidence and mortality rates. Cancer Epidemiol Biomarkers Prev. 2015;24(10): 1495-1506.
30. McPherson K, Steel CM, Dixon JM. ABC of breast diseases. Breast cancer-epidemiology, risk factors, and genetics. BMJ. 2000;321(7261): 624-628.

31. Sosnowska B, Mazidi M, Penson P, Gluba-Brzozka A, Rysz J, Banach M. The sirtuin family members SIRT1, SIRT3 and SIRT6: their role in vascular biology and atherogenesis. Atherosclerosis. 2017;265: 275-282.

32. Winnik S, Auwerx J, Sinclair DA, Matter CM. Protective effects of sirtuins in cardiovascular diseases: from bench to bedside. Eur Heart J. 2015;36(48):3404-3412.

33. Tang X, Shi L, Xie N, et al. SIRT7 antagonizes TGF-beta signaling and inhibits breast cancer metastasis. Nat Commun. 2017;8(1):318.

34. Ford E, Voit R, Liszt G, Magin C, Grummt I, Guarente L. Mammalian Sir2 homolog SIRT7 is an activator of RNA polymerase I transcription. Genes Dev. 2006;20(9):1075-1080.

35. Lai CC, Lin PM, Lin SF, et al. Altered expression of SIRT gene family in head and neck squamous cell carcinoma. Tumour Biol. 2013; 34(3):1847-1854.

36. Moran VA, Perera RJ, Khalil AM. Emerging functional and mechanistic paradigms of mammalian long non-coding RNAs. Nucleic Acids Res. 2012;40(14):6391-6400.

37. Bergmann JH, Spector DL. Long non-coding RNAs: modulators of nuclear structure and function. Curr Opin Cell Biol. 2014; 26:10-18.

38. Gutschner T, Diederichs S. The hallmarks of cancer: a long non-coding RNA point of view. RNA Biol. 2012;9(6):703-719.

39. Gainor JF, Shaw AT. Emerging paradigms in the development of resistance to tyrosine kinase inhibitors in lung cancer. J Clin Oncol. 2013; 31(31):3987-3996.

40. Zhang EB, Yin DD, Sun M, et al. P53-regulated long non-coding RNA TUG1 affects cell proliferation in human non-small cell lung cancer, partly through epigenetically regulating HOXB7 expression. Cell Death Dis. 2014;5:e1243.

41. Hauptman N, Glavac D. Long non-coding RNA in cancer. Int J Mol Sci. 2013;14(3):4655-4669.

42. Vikram R, Ramachandran R, Abdul KS. Functional significance of long non-coding RNAs in breast cancer. Breast Cancer. 2014;21(5): 515-521.

43. Liu C, Cao B, Liu N, Zhou Z, Yang G, Zhou P. Increased expression of long noncoding RNA RP11-62F24.2 in gastric cancer and its clinical significance. Clin Lab. 2017;63(9):1475-1479.

44. Li XL, Subramanian M, Jones MF, et al. Long noncoding RNA PURPL Suppresses basal p53 levels and promotes tumorigenicity in colorectal cancer. Cell Rep. 2017;20(10):2408-2423.

45. Kiran S, Oddi V, Ramakrishna G. Sirtuin 7 promotes cellular survival following genomic stress by attenuation of DNA damage, SAPK activation and p53 response. Exp Cell Res. 2015;331(1):123-141.

46. Malik S, Villanova L, Tanaka S, et al. SIRT7 inactivation reverses metastatic phenotypes in epithelial and mesenchymal tumors. Sci Rep. 2015;5:9841.

47. Kiran S, Chatterjee N, Singh S, Kaul SC, Wadhwa R, Ramakrishna G. Intracellular distribution of human SIRT7 and mapping of the nuclear/ nucleolar localization signal. FEBS J. 2013;280(14):3451-3466.

48. Mohrin M, Shin J, Liu Y, et al. Stem cell aging. A mitochondrial UPRmediated metabolic checkpoint regulates hematopoietic stem cell aging. Science. 2015;347(6228):1374-1377.

49. Adhikari VP, Lu LJ, Kong LQ. Does hepatitis B virus infection cause breast cancer? Chin Clin Oncol. 2016;5(6):81.

50. Yang F, Zhang L, Huo XS, et al. Long noncoding RNA high expression in hepatocellular carcinoma facilitates tumor growth through enhancer of zeste homolog 2 in humans. Hepatology. 2011;54(5):1679-1689.

51. Rupaimoole R, Lee J, Haemmerle M, et al. Long noncoding RNA ceruloplasmin promotes cancer growth by altering glycolysis. Cell Rep. 2015;13(11):2395-2402.

52. Wang Q, Tan YX, Ren YB, et al. Zinc finger protein ZBTB20 expression is increased in hepatocellular carcinoma and associated with poor prognosis. BMC Cancer. 2011;11:271. 


\section{Supplementary materials}

Table SI Characteristics of breast cancer cell line of MDA-MB-23I and MCF-7

\begin{tabular}{lll}
\hline Cell line & MDA-MB-23 I & MCF-7 \\
\hline Organism & Homo sapiens, human & Homo sapiens, human \\
Tissue & Mammary gland/breast; derived from & Mammary gland, breast; derived from \\
& metastatic site: pleural effusion & metastatic site: pleural effusion \\
Cell type & Epithelial & Epithelial \\
Disease & Adenocarcinoma & Adenocarcinoma \\
Age & $5 \mathrm{I}$-year-old adult & $69-$ year-old adult \\
Gender & Female & Female \\
Applications & These cells are a suitable transfection host & These cells are a suitable transfection host \\
\hline
\end{tabular}

Table S2 siRNA sequences of SIRT7 that were used in this study

\begin{tabular}{|c|c|c|}
\hline$\overline{\text { Genes }}$ & Forward & Reverse \\
\hline siSIRT7-I & 5'-GGAAGUGUGAUGACGUCAUTT-3' & 5'-AUGACGUCAUCACACUUCCTT-3' \\
\hline siSIRT7-2 & 5'-GCCAAAUACUUGGUCGUCUTT-3' & 5'-AGACGACCAAGUAUUUGGCTT-3' \\
\hline siSIRT7-3 & 5'-CACCGUAUUUCUACUACUATT-3' & 5'-UAGUAGUAGAAAUACGGUGTT-3' \\
\hline Negative control & 5'-UUCUCCGAACGUGUCACGUTT-3' & 5'-ACGUGACACGUUCGGAGAATT-3' \\
\hline
\end{tabular}

Table S3 Primer sequences of IncRNA and mRNA that were determined in this study

\begin{tabular}{|c|c|c|}
\hline Genes & Forward & Reverse \\
\hline LINCOI002 & 5'-CCCTCCGATGGCATCTCCAG-3' & 5'-GGAGAGCCCGACTTCAGGAC-3' \\
\hline LINC00630 & 5'-AAGGCTCTGGTGAGAAACCCA-3' & 5'-TGCATGAAGAGTTGCACAGGGA-3' \\
\hline RPII-I60E2.6 & 5'-GCGATCTCGGCTCCTAGCAA-3' & 5'-GCTACTCGGGAGGGCTTAGG-3' \\
\hline LIPE-ASI & 5'-GCCCAAGAGCGGAGGTAGAG-3' & 5'-AAGGCGGTCCAGAAGCAGTT-3' \\
\hline $\mathrm{RCHYI}$ & 5'-GTCTCCTAAAGGCACCTTGC-3' & 5'-ATGCACTGCACTTCCTTCAC-3' \\
\hline ATF2 & 5'-TCAGCAGGCAGTACCTTCAC-3' & 5'-AGGGACAATTGGCCTGTTAG-3' \\
\hline CCNG2 & 5'-GAGGCTCACAGACAAGTCCA-3' & 5'-CATCACAGTCATGGGAAAGC-3' \\
\hline E2F2 & 5'-TCAAGCACCTGACTGAGGAC-3' & 5'-CCTTAAAGTTGCCAACAGCA-3' \\
\hline HSPBI & 5'-GTCCCTGGATGTCAACCACT-3' & 5'-CACGCCATCCTTGGTCTT-3' \\
\hline$\beta$-Actin & 5'-AGGTCATCACTATTGGCAAC-3' & 5'-ACTCATCGTACTCCTGCTTG-3' \\
\hline
\end{tabular}

\section{Publish your work in this journal}

OncoTargets and Therapy is an international, peer-reviewed, open access journal focusing on the pathological basis of all cancers, potential targets for therapy and treatment protocols employed to improve the management of cancer patients. The journal also focuses on the impact of management programs and new therapeutic agents and protocols on

\section{Dovepress}

patient perspectives such as quality of life, adherence and satisfaction. The manuscript management system is completely online and includes a very quick and fair peer-review system, which is all easy to use. Visit http://www.dovepress.com/testimonials.php to read real quotes from published authors. 